\title{
Foreword to the Special Issue on the Frontiers of IEMS in Asia-Pacific Area
}

\author{
Jin Peng* \\ Institute of Uncertain Systems, Huanggang Normal University, Hubei, China \\ Takashi Irohara \\ Department of Information and Communication Sciences, Faculty of Science and Technology, \\ Sophia University, Tokyo, Japan
}

* Corresponding Author, E-mail: pengjin01@tsinghua.org.cn

The 12th Asia Pacific Industrial Engineering and Management Systems Conference (APIEMS2011) was held in Beijing, the cultural, educational, and political capital of China on October 14-16, 2011. This conference successfully provided a forum for exchanging ideas on the latest developments in the field of industrial engineering and management systems among researchers and engineers working in universities and industries, and created opportunities for collaboration among the participants.

This special issue comprises of papers which cover the topics related to the frontiers of industrial engineering and management systems in the Asia-Pacific area selected from APIEMS 2011. For this second special issue, 8 papers were finally accepted after the normal reviewing process.

The first paper by Nagasawa et al. applies the canonical correlation and cluster analysis methodology to study ordering system selection and items classification. The second paper by Takezawa et al. builds bridges between facialbased or situation-based emotions and prosocial behaviors by a computer-based training program. The third paper by Seki et al. uses the multinomial logit model to evaluate the spread of micro electric vehicle (EV) within a local community. The fourth paper by Mak et al. proposes an improved genetic algorithm to maximize the manufacturer's total profit subject to various operating constraints of the manufacturing system. The fifth paper by Tseng and Chiu evaluates a firm's green supply chain management under incomplete information condition. The sixth paper by Suzuki and Yamamoto offers solutions to the facility rearrangement problem by applying a genetic algorithm and heuristic local search. The seventh paper by $\mathrm{Li}$ and Peng proposes a comparison method for uncertain risks. The last paper by Sheng and Yao investigates the fixed charge transportation problem in uncertain environment and presents an uncertain programming model.

As guest editors for this special issue, we would like to thank the editor-in-chief of IEMS, Prof. Mitsuo Gen, for giving us the opportunity to publish selected papers as a special issue of the journal. Special thanks to the managing editor, Prof. Chi-Hyuck Jun, for his time and effort in processing and managing the manuscripts. In addition, we would like to thank all the peer reviewers for reviewing the special issue papers. 